with AC patients (MAC: sub-distribution hazard ratio (SHR), 0.54 ; 95\% CI. 0.13-2.22, $\mathrm{p}=0.397$ and SRC: SHR, 0.87; $95 \% \mathrm{CI}, 0.62-1.23, \mathrm{p}=0.435)$ in the multivariate competing risk model. When MAC and SRC patients were further compared to AC patients with grade III/IV, LNM and survival were still not correlated with histology.

Conclusions Early gastric MAC and SRC had similar behaviour compared with AC in Western population and histology itself is not an independent predictor of LNM and prognosis.

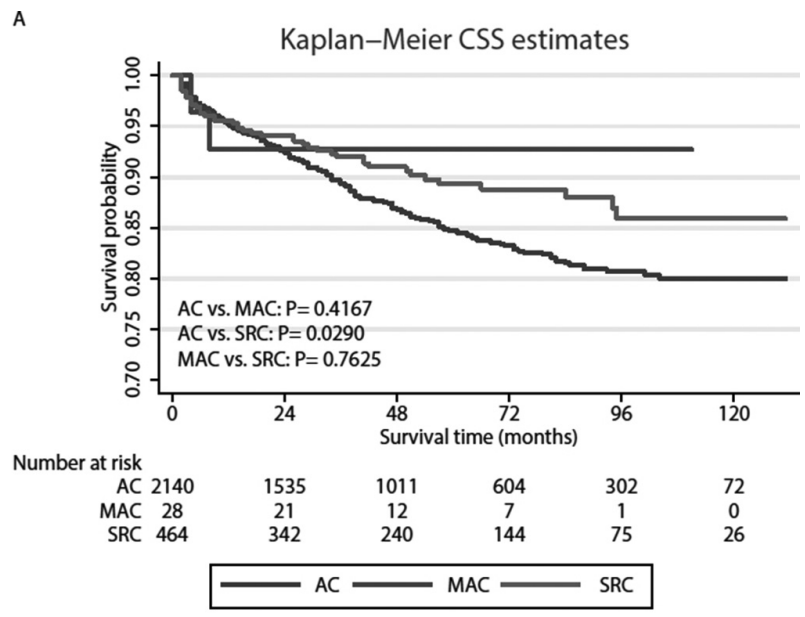

Abstract IDDF2018-ABS-0127 Figure 1 Kaplan-Meier CSS estimates

\section{IDDF2018-ABS-0128 EFFECTS OF STOOL FORM ON ADEQUACY OF BOWEL PREPARATION DURING COLONOSCOPY: A PROSPECTIVE STUDY}

Mark Lester Sy*, Rei Joseph Prieto, Alex Pang Jr, Michael Angelo Chu, Lovell Gatchalian, Felix Domingo, Marichona Naval. East Avenue Medical Center, Philippines

\subsection{6/gutjin-2018-IDDFabstracts. 117}

Background Early identification of adenomatous polyps is crucial in colorectal cancer prevention. Inadequate bowel preparation results in lower diagnostic yield and is affected by colonic transit. There are insufficient local studies correlating stool form with bowel preparation adequacy. Moreover, there's limited study correlating stool form with colonoscopy outcome. This study aims to test the association between Bristol Stool Form Scale (BSFS), bowel preparation adequacy and colonoscopy outcome.

Methods We studied a prospective cohort of 260 consecutive outpatients at East Avenue Medical Centre (EAMC) undergoing screening colonoscopy who were prescribed similar bowel preparation and diet. BSFS and comorbidities were collected prior to colonoscopy. Patients were grouped according to BSFS: Group 1 for type 1-2; Group 2 for type 3-5; Group 3 for type 6-7. An investigator, blinded to all information, recorded Boston Bowel Preparation Score (BBPS), Polyp detection rate (PDR), cecal intubation and withdrew time for all patients. Chi-Square, ANOVA and logistic regression were the analysis used.

Results 260 were included in the analysis. Group 1 compared to Groups 2 and 3, was significantly associated with inadequate bowel preparation $(24.3 \%$ vs $0 \%$ vs $9.1 \%$; $=0.004)$, low cecal intubation rate $(87 \%$ vs $100 \%$ vs $97 \%$; p $<0.001)$, low PDR ( $11 \%$ vs $32 \%$ vs $24 \%$; $=0.004)$, and prolonged withdrawal time $(9.41 \mathrm{~min} ; \mathrm{p}<0.001)$. Higher BSFS score $(3-$ 7 ) would have $157 \%$ higher odds of adequate bowel preparation. Diabetes have a likelihood of only $24 \%$ adequate bowel preparation.

Conclusions It's important to identify patients with constipated stool form to guide proper bowel preparation regimen since they are significantly associated with inadequate bowel preparation, decreased cecal intubation rate, PDR, and increased withdrawal time.

\section{IDDF2018-ABS-0129 EFFECTIVENESS OF REBAMIPIDE IN THE PREVENTION OF ESOPHAGEAL STRICTURE FORMATION IN ADVANCED CORROSIVE ESOPHAGITIS: A PROSPECTIVE RANDOMISED CONTROL STUDY}

Mark Lester Sy*, Rei Joseph Prieto, Alex Pang Jr, Michael Angelo Chu, Lovell Gatchalian, Gerby Coronel, Felix Domingo, Marichona Naval. East Avenue Medical Center, Philippines

\subsection{6/gutjnl-2018-IDDFabstracts. 118}

Background Corrosive esophagitis causes significant morbidity with grades $2 \mathrm{~b}$ and 3 esophagitis developing strictures in $70 \%-$ $100 \%$ of cases. Currently, there's no clear evidence in preventing esophageal stricture. However, animal studies show that suppressing inflammation can prevent stricture formation. Rebamipide, an anti-ulcer agent, has been shown to stimulate prostaglandin generation, increases epidermal growth factor expression, increases blood flow, and scavenges active oxygen radicals thereby reducing inflammation. This study aims to determine the effectiveness of rebamipide in preventing stricture formation in advanced corrosive esophagitis.

Methods We conducted a prospective, assessor-blinded, randomised control study. 37 adult patients with grades 2B-3B corrosive esophagitis admitted in our institution were included. Patients were randomly allocated to two groups. The first group $(n=18)$ received conventional proton pump inhibitor (PPI) therapy with rebamipide (PPI+rebamipide group) while the second group $(n=19)$ received only conventional PPI therapy (PPI group), both for 3 weeks duration. Endoscopists blinded to group allocations and damage severity before treatment performed upper endoscopy (EGD) on Days 21 and 60. Presence of dysphagia, number, length and stricture location were recorded. Descriptive statistics, Independent sample Ttest, Mann-Whitney $\mathrm{U}$ test and Chi-square test were the analysis used.

Results 34 patients were included in the final analysis. Dysphagia and esophageal stricture at Day 60 were significantly greater with PPI group versus PPI+rebamipide group (47\% vs $5.88 \%, \mathrm{p}=0.017)$. The median length of esophageal stricture in the PPI group at Day 60 is significantly shorter $(5 \mathrm{~cm}$ vs $8 \mathrm{~cm}$; $\mathrm{p}=0.034$ ) however there were still significantly more patients with stricture formation in the PPI group (8 vs 1; $\mathrm{p}=0.017)$. Presence of gastric stricture was similar between the two groups.

Conclusions PPI with rebamipide is effective in preventing dysphagia and stricture formation among patients with advanced corrosive esophagitis compared to PPI alone. However, this was not effective in preventing gastric stricture formation. 